\title{
EDITORIAL
}

\section{How much is too much? The treatment of mild asthma}

\author{
P.M. O’Byrne
}

$\mathbf{M}$ ild persistent asthmatic patients constitute a significant proportion of patients (up to $70 \%$ of all asthmatics) [1]. These patients might be considered the "silent majority" of asthmatics. This is because they rarely visit their primary care physician with symptoms of asthma, and are even more rarely seen in a secondary or tertiary healthcare setting, where physicians with a focused interest in asthma management tend to work. It is partly for this reason that, until recently, very little attention has been paid to the morbidity associated with mild persistent asthma and very few studies have evaluated the responses of this patient population to treatment.

Asthma management guidelines typically describe mild persistent asthma as patients having: asthma symptoms more than weekly, but less than daily, and nocturnal symptoms less than weekly, with normal lung function (forced expired volume in one second (FEV1) or peak expiratory flow $>80 \%$ ) between asthma episodes [2]. However, most studies that claim to have studied patients with "mild-to-moderate asthma" have not included many (or any) patients with mild asthma, as reflected by the mean FEV1, which was usually in the range of $70 \%$ predicted normal and/or rescue $\beta_{2}$-agonist use of 2-3 puffs day $^{-1}$ [3-5]. This makes these studies of little or no value in deciding on treatment strategies for patients with mild persistent asthma. However, there has been an increase in the number of studies focusing on the optimal management of mild persistent asthma, some of which have been published very recently.

\section{CURRENT TREATMENT RECOMMENDATIONS FOR MILD PERSISTENT ASTHMA}

Asthma treatment guidelines have been consistent in describing the goals and objectives of asthma treatment. These are to: 1) minimise or eliminate asthma symptoms; 2 ) achieve the best possible lung function; 3) prevent asthma exacerbations; 4) do the above with the least possible medications; 5) minimise short- and long-term adverse effects; and 6) educate the patient about the disease and the goals of management [2]. In addition to these goals and objectives, each of these documents has described what is meant by the term "asthma control". This includes the objectives above but also includes minimising the need for rescue medications, such as inhaled $\beta_{2}$-agonists to less than daily use, minimising the variability of flow rates that is

\section{STATEMENT OF INTEREST: None declared.}

CORRESPONDENCE: P.M. O'Byrne, Firestone Institute for Respiratory Health, St. Joseph's Healthcare and Dept of Medicine, McMaster University Medical Center, 1200 Main Street West, Hamilton, ON, L9G 4R7, Canada. Fax: 1 9055214973. E-mail: obyrnep@mcmaster.ca characteristic of asthma, as well as having normal activities of daily living. Some of these outcomes can be regarded as "patient centred", which are outcomes that the patient would regard as obvious benefits of treatment, such as eliminating asthma symptoms, preventing severe exacerbations, normal activities of daily living and minimising medication adverse effects. Other outcomes can be considered as "physician centred", particularly measurements of lung function, which are very important in establishing asthma control but changes in which are often not recognised by the patient. This distinction is particularly important in studies of mild asthma, where lung function is already (near) normal.

A number of studies have examined the benefits of inhaled corticosteroids (ICS), which are the pre-eminent anti-inflammatory therapy in asthma, in patients with mild persistent asthma. The conclusion reached from these studies has been controversial. The first study published was the OPTions In Management with Antiretrovirals (OPTIMA) trial [6]. In this trial, two groups of patients were selected. One group consisted of almost 700 ICS-naïve patients, in which the ICS alone (budesonide $200 \mu \mathrm{g} \cdot \mathrm{day}^{-1}$ ) was compared to the same dose of budesonide plus the long-acting $\beta_{2}$-agonist (LABA) formoterol or placebo for $1 \mathrm{yr}$ of treatment. The primary outcome variable in the study was the rate of severe asthma exacerbations. The rate of severe asthma exacerbations was higher than expected in mild patients, being 0.77 severe exacerbations per patient per year in the placebo treated group. This rate was reduced to 0.26 per patient per year during treatment with low-dose budesonide alone. All other outcomes, including days with asthma symptoms and nights with nocturnal symptoms, were improved by budesonide treatment. The combination of budesonide and formoterol did not provide any additional benefit when compared to budesonide alone.

The second large study evaluating ICS in mild persistent asthma was the Steroid Treatment As Regular Therapy (START) trial [7]. This study examined whether early intervention with ICS (budesonide) prevented the progression of asthma, in both adults and children, with newly diagnosed mild persistent asthma as measured by the time to the first very severe exacerbation, which required an emergency room visit or hospitalisation, and by the decline in post-bronchodilator FEV1. The patients were treated with low-dose budesonide or placebo for $3 \mathrm{yrs}$. By the end of the double-blind treatment period, $50 \%$ of placebo-treated patients, who had not been on an ICS at the start of the study, were being treated with ICS and $6 \%$ of the population had a severe asthma exacerbation. In the budesonide group, $30 \%$ of the patients were given additional ICS treatment and $3 \%$ of the population 
had a severe asthma exacerbation. The changes in both preand post-bronchodilator FEV1, while statistically significant, were small at the end of 3 yrs of treatment. Thus, early intervention with low doses of ICS did prevent the progression of asthma but the effects were incomplete, particularly in the case of lung function.

A third large prospective trial of ICS compared with nedocromil or placebo was the Childhood Asthma Management Plan (CAMP) study [8]. This study was designed to evaluate whether treatment with either low-dose ICS (budesonide $200 \mathrm{mcg}$ ) or nedocromil $(8 \mathrm{mg}$ ) prevented the decline in lung function in asthmatic children. The primary outcome variable was change in the post-bronchodilator FEV1 $\%$ pred, and this was not significantly improved by either active treatment. However, the children who received budesonide had a significantly smaller decline in the ratio of FEV1 to forced vital capacity, as well as improved airway responsiveness to methacholine, fewer hospitalisations, fewer urgent visits to a caregiver, a greater reduction in the need for rescue treatment for symptoms, fewer courses of prednisone, and fewer days requiring additional asthma medications.

The IMProving Asthma Control Trial (IMPACT) trial [9] evaluated intermittent short-course corticosteroid treatment, guided by a symptom-based action plan alone or in addition to daily treatment with either ICS, budesonide, or the antileukotriene zafirlukast for $1 \mathrm{yr}$, in 225 patients with mild persistent asthma. This patient population was almost identical in baseline characteristics to the other two larger studies, albeit with a much longer duration of asthma than in the other studies. The authors concluded from the results of the study that it may be possible for patients with mild persistent asthma to be treated with intermittent courses of inhaled or oral corticosteroids, together with an action plan. This conclusion was reached despite the fact that regular use of inhaled budesonide was significantly better than intermittent use in improving pre-bronchodilator FEV1, asthma control scores, number of symptom-free days, airway hyperresponsiveness and markers of airway inflammation.

The literature on the management of mild persistent asthma has been increased by two recent studies that addressed two other important issues in this patient population. The first of these evaluated whether the symptom-driven use of a combination inhaler containing the ICS beclomethasone dipropionate (BDP) and the short-acting inhaled $\beta_{2}$-agonist salbutamol is as effective as the regular use of inhaled BDP [10]. The study was a 6-month, double-blind, randomised trial in 455 patients with mild persistent asthma. The primary outcome was the morning peak expiratory flow (PEF) rate. The $\mathrm{PEF}$ rate and the number of exacerbations were not significantly different in the as-needed combination-therapy group compared with those in the groups receiving regular BDP or regular combination therapy, but were significantly better than the as-needed salbutamol-alone group. In addition, the cumulative dose of inhaled BDP was significantly lower in the as-needed combination-therapy group than in the groups receiving regular BDP therapy or regular combination therapy. The mean cumulative doses of BDP over the 6 months of treatment were $77 \mathrm{mg}$ (an average of $500 \mathrm{mcg} \cdot$ day $^{-1}$ ) in the regular $\mathrm{BDP}$ and the regular combination therapy arms, and
$18.5 \mathrm{mg}$ (an average of $120 \mathrm{mcg} \cdot \mathrm{day}^{-1}$ ) in the as-needed combination therapy arm.

The fact that treatment with regular BDP alone or as-needed BDP and salbutamol was significantly better in reducing asthma exacerbations than either arm that contained regular salbutamol use raises a number of interesting issues. The first of these is that any treatment option that contains regular salbutamol treatment should be avoided in mild asthma. Secondly, the benefit in reducing exacerbations required ICS, but a low dose may be needed to achieve this benefit. This is consistent with the other studies which have addressed this question in mild persistent asthma, which have used budesonide $200 \mathrm{mcg}^{\circ}$ day $^{-1}$ (albeit with regular administration) and which have shown a similar degree of benefit in reducing exacerbations [6, 7]. This low dose may be particularly beneficial when administered at times when symptoms are increasing and airway inflammation worsening as an exacerbation develops, as is suggested by the efficacy of maintenance and rescue use of the combination ICS containing budesonide and formoterol in reducing severe asthma exacerbations in more severe asthma $[11,12]$.

A second recent study evaluated a number of possible strategies in reducing treatment in patients with mild persistent asthma whose asthma was well controlled on regular ICS therapy [13]. The study randomised 500 patients with well-controlled asthma while on ICS therapy (fluticasone $100 \mathrm{mcg}$ twice daily) to receive continued fluticasone (100 mcg twice daily), the leukotriene antagonist montelukast once daily, or the combination of fluticasone $(100 \mathrm{mcg})$ and the LABA salmeterol once daily for 16 weeks. The primary outcome was the time to treatment failure. The study demonstrated that patients treated with continued fluticasone or fluticasone and salmeterol had significantly fewer treatment failures when compared to subjects treated with montelukast (20 versus $30.3 \%$ ). The authors concluded that if asthma is well controlled with the use of twice-daily inhaled fluticasone treatment can be switched to once-daily fluticasone and salmeterol without increased rates of treatment failure. This conclusion, however, is limited by the fact that the study did not contain a treatment arm with fluticasone alone once daily. As the dose response to ICS is very steep for most clinical outcomes [14], and no studies have been able to demonstrate an important clinical difference with doubling or halving the maintenance dose of ICS in patients with mild persistent asthma [6], it is uncertain whether the addition of salmeterol provided any useful clinical benefit when compared to lowdose fluticasone alone.

\section{IS IT WORTH USING REGULAR ICS IN MILD PERSISTENT ASTHMA?}

The most widely used primary-outcome variables in asthma efficacy trials (improvements in FEV1 or morning PEF) are not the best choice in studies of patients with mild persistent asthma. This is because, by definition, these measurements will be normal or close to normal most of the time in this patient population. Even using asthma symptom scores, asthma control questionnaires or asthma quality of life tools, which are better options, requires large sample sizes to be adequately powered because of the small magnitude of change likely to be seen, as these patients are asymptomatic much of the time. 
Patients with mild persistent asthma, have, by definition, persistent symptoms weekly, with nocturnal symptoms occasionally and rates of severe asthma exacerbations, if untreated, which are higher than expected in two out of the three studies which have examined this $[6,7]$. It is also well established that many asthmatic patients with persistent asthma minimise symptoms or come to accept that they are part of everyday life [15]. Efficacy can be demonstrated in all studies that have evaluated ICS in mild persistent asthma; therefore, a therapeutic trial of low doses of ICS (which should probably be for a minimum of 3 months to obtain most of the therapeutic benefit [16]) in patients with mild persistent asthma may provide the magnitude of benefit that will ensure that patients will continue to use the medication. If this benefit is not achieved, it is unlikely that the patient will continue to use ICS on a regular basis and will revert to using the medication intermittently. However, if a therapeutic trial is not attempted, the magnitude of the clinical effectiveness will never be known. The recent study by PAPI et al. [10] provides an interesting alternative to regular daily ICS, whereby patients use a combination inhaler of ICS and LABA as needed. It is clear from this study, however, that some ICS is needed to reduce the risk of exacerbations, but the dose used was $\sim 20 \%$ of the regular maintenance dose in that study. Once again, this emphasises the very steep dose-response of ICS in mild asthma, with most of the benefits achieved at low doses.

The other issue that needs to be considered when making a decision to start ICS treatment in mild asthma is the potential for side-effects. ICS are not metabolised in the lungs and every molecule of ICS that is administered into the lungs is absorbed into the systemic circulation. All of the studies in patients with mild persistent asthma have used maximal doses of $500 \mathrm{mcg} \cdot \mathrm{day}^{-1}$. There is a wealth of data demonstrating the safety of these low doses in adults, even when used long term [16]. However, in both the START trial [7] and the CAMP study [8] there was a significant reduction in growth velocity in the children in the study of $1.0-1.5 \mathrm{~cm}$ over the $3-5 \mathrm{yrs}$ of treatment with budesonide $200-400 \mathrm{mcg} \cdot \mathrm{day}^{-1}$. This is unlikely to have any effect on the final height of these children, as the only study that has followed children treated with inhaled budesonide to final height did not show any detrimental effect,

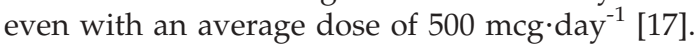

\section{CONCLUSION}

Mild intermittent and persistent asthma constitutes the majority of asthmatic patients. The choice of measurements of change in lung function as the primary outcome variable (as is often done in clinical trials of more severe asthmatic populations) is often inappropriate when studying mild persistent asthma. The currently available evidence indicates that low doses of inhaled corticosteroids ( $<400 \mathrm{mcg}$ of beclomethasone dipropionate or equivalent) can often provide an ideal asthma control and reduce the risks of severe asthma exacerbations in both children and adults with mild persistent asthma, and should be the treatment of choice. There is no convincing evidence that regular use of combination therapy with inhaled corticosteroids and long-acting $\beta_{2}$-agonists provides any additional benefit. Intermittent inhaled corticosteroid therapy at the time of an exacerbation has also been suggested to be an effective treatment strategy for mild persistent asthma, but for most important outcomes it is less effective than low-dose regular therapy. The "as-needed" use of inhaled corticosteroids (together with an inhaled $\beta_{2}$-agonist) has shown promise in one study, but this needs to be replicated with other combinations of inhaled corticosteroids and inhaled $\beta_{2}$-agonists, as the combination used in this study (a first-generation inhaled corticosteroid, beclomethasone dipropionate and a short-acting and inhaled $\beta_{2}$-agonist, salbutamol) has not been extensively studied in other settings and is unlikely to be widely available for use. Leukotriene antagonists are another treatment option in this population but they are also less effective than low-dose inhaled corticosteroids.

\section{REFERENCES}

1 Kwok MY, Walsh-Kelly CM, Gorelick MH, Grabowski L, Kelly KJ. National Asthma Education and Prevention Program severity classification as a measure of disease burden in children with acute asthma. Pediatrics 2006; 117: S71-S77.

2 Global Initiative for Asthma. Global strategy for asthma management and prevention. Bethesda, National Heart, Lung and Blood Institute, 2006.

3 Pearlman DS, Berger WE, Kerwin E, Laforce C, Kundu S, Banerji D. Once-daily ciclesonide improves lung function and is well tolerated by patients with mild-to-moderate persistent asthma. J Allergy Clin Immunol 2005; 116: 1206-1212.

4 Pearlman DS, Peden D, Condemi JJ, et al. Efficacy and safety of fluticasone propionate/salmeterol HFA 134A MDI in patients with mild-to-moderate persistent asthma. J Asthma 2004; 41: 797-806.

5 Israel E, Rubin P, Kemp JP, et al. The effect of inhibition of 5-lipoxygenase by zileuton in mild-to-moderate asthma. Ann Intern Med 1993; 119: 1059-1066.

6 O’Byrne PM, Barnes PJ, Rodriguez-Roisin R, et al. Low dose inhaled budesonide and formoterol in mild persistent asthma: the OPTIMA randomized trial. Am J Respir Crit Care Med 2001; 164: 1392-1397.

7 Pauwels RA, Pedersen S, Busse WW, et al. Early intervention with budesonide in mild persistent asthma: a randomised, double-blind trial. Lancet 2003; 361: 1071-1076.

8 Long-term effects of budesonide or nedocromil in children with asthma, The Childhood Asthma Management Program Research Group. N Engl J Med 2000; 343 1054-1063.

9 Boushey HA, Sorkness CA, King TS, et al. Daily versus asneeded corticosteroids for mild persistent asthma. $N$ Engl J Med 2005; 352: 1519-1528.

10 Papi A, Canonica GW, Maestrelli P, et al. Rescue use of beclomethasone and albuterol in a single inhaler for mild asthma. N Engl J Med 2007; 356: 2040-2052.

11 O'Byrne PM, Bisgaard H, Godard PP, et al. Budesonide/ formoterol combination therapy as both maintenance and reliever medication in asthma. Am J Respir Crit Care Med 2005; 171: 129-136.

12 Rabe KF, Atienza T, Magyar P, Larsson P, Jorup C, Lalloo UG. Effect of budesonide in combination with formoterol for reliever therapy in asthma exacerbations: a randomised controlled, double-blind study. Lancet 2006; 368: 744-753. 
13 Peters SP, Anthonisen N, Castro M, et al. Randomized comparison of strategies for reducing treatment in mild persistent asthma. N Engl J Med 2007; 356: 2027-2039.

14 Pedersen S, O'Byrne PM. A comparison of the efficacy and safety of inhaled corticosteroids in asthma. Allergy 1997; 52: 1-34.

15 Rabe KF, Adachi M, Lai CK, et al. Worldwide severity and control of asthma in children and adults: the global asthma insights and reality surveys. J Allergy Clin Immunol 2004; 114: 40-47.

16 Barnes PJ, Pedersen S, Busse WW. Efficacy and safety of inhaled corticosteroids. New developments. Am J Respir Crit Care Med 1998; 157: S1-53.

17 Agertoft L, Pedersen S. Effect of long-term treatment with inhaled budesonide on adult height in children with asthma. N Engl J Med 2000; 343: 1064-1069. 